\title{
Theoretical study on threshold energy and impact ionization coefficient for electrons in $\mathrm{Si}_{1-x} \mathbf{G e}_{x}$
}

\author{
Keesoo Yeom, John M. Hinckley, and Jasprit Singh \\ Department of Electrical Engineering and Computer Science, The University of Michigan, Ann Arbor; \\ Michigan 48109-2122
}

(Received 13 December 1993; accepted for publication 13 March 1994)

\begin{abstract}
Threshold energy and electron impact ionization coefficients $(\alpha)$ are calculated for unstrained and strained $\mathrm{Si}_{1-x} \mathrm{Ge}_{x}$ on $\{100\}$ silicon substrate using nonparabolic and ellipsoidal band structure for conduction band and $\mathbf{k} \cdot \mathbf{p}$ method for valence band. The threshold energy in the unstrained $\mathrm{Si}_{1-x} \mathrm{Ge}_{x}$ is smaller than that in pure silicon due to the reduced band-gap energy. The strain causes band degeneracy lifting for both the conduction band and valence band. It gives an additional band-gap narrowing which leads to a much smaller threshold energy. On the basis of these results, the electron impact ionization coefficient is estimated up to $30 \%$ germanium using a Monte Carlo simulation. The reduced threshold energy is found to be the most dominant factor in determining $\alpha$ in the strained $\mathrm{Si}_{1-x} \mathrm{Ge}_{x}$. As a result, the strained $\mathrm{Si}_{1-x} \mathrm{Ge}_{x}$ has much larger $\alpha$ than pure silicon while the unstrained $\mathrm{Si}_{1-x} \mathrm{Ge}_{x}$ does not due to the effect of alloy scattering and the relatively small change of the threshold energy.
\end{abstract}

The $\mathrm{Si}_{1-x} \mathrm{Ge}_{x}$ alloy is emerging as a very important material system for applications in both electronics and optoelectronics. The $\mathrm{Si} / \mathrm{Si}_{1-x} \mathrm{Ge}_{x} \mathrm{HBT}^{1,2}$ and $\mathrm{Si}_{1-x} \mathrm{Ge}_{x}$ avalanche photodiode are two important devices that may benefit from this technology. In both these devices, it is important to understand the impact ionization breakdown initiated by electrons. While several transport studies on $\mathrm{Si}_{1-x} \mathrm{Ge}_{x}$ alloys have been reported in the literature, ${ }^{3-5}$ there has been no work on the impact ionization coefficient. In this letter, we focus on the electron breakdown in $\mathrm{Si}_{1-x} \mathrm{Ge}_{x}$ alloys and report theoretical results for the electron impact ionization coefficient $(\alpha)$ for a variety of unstrained and strained alloy compositions.

The first step in estimating the impact ionization coeffcient is to find the threshold energy for impact ionization. At the threshold condition, energy and momentum conservation should be satisfied. The total energy $E_{f}$ and the total momentum of the resultant carriers $\mathbf{K}_{f}$ are given by

$$
E_{f}=E_{c}\left(\mathbf{k}_{1}\right)+E_{c}\left(\mathbf{k}_{2}\right)-E_{\nu}\left(\mathbf{k}_{3}\right)
$$

and

$$
\mathbf{K}_{f}=\mathbf{k}_{1}+\mathbf{k}_{2}-\mathbf{k}_{3},
$$

where $E_{c}(\mathbf{k})$ and $E_{\nu}(\mathbf{k})$ are energy of carriers at $\mathbf{k}$ in the conduction band and valence band, respectively. To minimize the total energy $E_{f}$ for a given momentum $\mathbf{K}_{f}$, the final carriers should have the same group velocity. ${ }^{6}$ With this condition and the given band structure, the threshold energy can be found.

We will consider two cases in this letter: (i) the $\mathrm{Si}_{1-x} \mathrm{Ge}_{x}$ alloy is unstrained. This situation would arise if a thick (several micron) layer of $\mathrm{Si}_{1-x} \mathrm{Ge}_{x}$ was grown on a silicon substrate; (ii) the $\mathrm{Si}_{1-x} \mathrm{Ge}_{x}$ alloy is pseudomorphically matched to $\{100\}$ silicon substrate. In this case, the thickness of the alloy would be below the critical thickness which depends upon the germanium mole fraction.

In the unstrained alloy, the conduction band valleys are described by a nonparabolic ellipsoidal band structure given by

$$
\gamma(E)=E(1+\alpha E)=\frac{\hbar^{2}}{2}\left(\frac{k_{l}^{2}}{m_{l}}+2 \frac{k_{t}^{2}}{m_{t}}\right) .
$$

The character of the valleys remains Si-like up to a Ge mole fraction of $85 \% .^{7}$ The conduction band effective masses $m_{l}$ and $m_{t}$ are obtained by interpolation for the alloy.

In the presence of strain, the degeneracy of the valleys is lifted as follows: in (001), (001) valleys

$$
\Delta E=\Xi_{d}^{(100)}\left(\epsilon_{x x}+\epsilon_{y y}+\epsilon_{z z}\right)+\Xi_{u}^{(100)} \epsilon_{z z}
$$

and in (100), (100), (010), (0ī0) valleys

$$
\Delta E=\Xi_{d}^{(100)}\left(\epsilon_{x x}+\epsilon_{y y}+\epsilon_{z z}\right)+\Xi_{u}^{(100)} \epsilon_{x x},
$$

where $\Xi_{d}^{(100)}$ and $\Xi{ }_{u}^{(100)}$ are the dilation and uniaxial deformation potential for the conduction band (100) valley and $\epsilon$ is strain of the material.

The valence band is determined by a six-band $\mathbf{k} \cdot \mathbf{p}$ Hamiltonian ${ }^{8}$ in which the effect of the strain can be included via the deformation potential theory. The effect of the strain is to lift the $\mathrm{HH}$ and $\mathrm{LH}$ degeneracy at $k=0$ and also to reduce the effective hole masses. The band-gap change by the degeneracy lifting of the conduction band and the valence band is shown in Fig. 1 by the lines (solid lines for the unstrained system, dashed lines for the strained system).

Having established the band structure, the threshold energy for impact ionization can be calculated. Table 1 represents the results for pure silicon and pure germanium with band-gap energy used in the calculation. The data in parenthesis are the values from Ref. 6. The threshold energy in silicon is almost the same as the band-gap energy. In addition, there is no threshold condition for electrons along the $\langle 111\rangle$ in silicon. On the other hand, germanium has a threshold condition in both $\langle 100\rangle$ and $\langle 111\rangle$ directions and the threshold energy is slightly larger $(0.1-0.2 \mathrm{eV})$ than band gap in $\langle 111\rangle$ direction. Figure 1 shows the threshold energy results for the unstrained and the strained $\mathrm{Si}_{1-x} \mathrm{Ge}_{x}$, respectively. The lines are band-gap energies from the valence band 


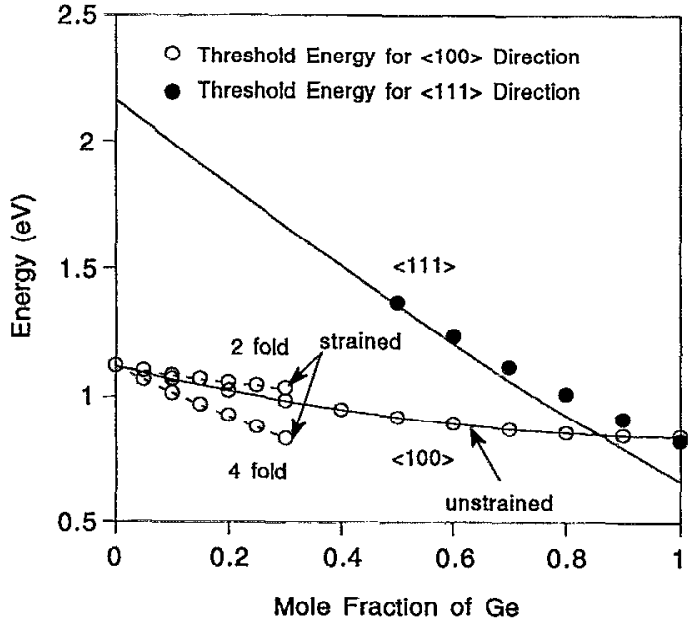

FIG. 1. Threshold energy for electron impact ionization in unstrained and strained $\mathrm{Si}_{1-x} \mathrm{Ge}_{x}$ on $\{100\}$ silicon substrate. Solid lines are the $X$ valley and $L$ valley hand gaps in unstrained $\mathrm{Si}_{1-x} \mathrm{Ge}_{x}$ and dashed lines represent the band gaps for the strained $\mathrm{Si}_{1-x} \mathrm{Ge}_{x}$ at the (001) (001) valleys (twofold) and (100) (100) (010) (0ï) valleys (fourfold). Marks are the threshold energy from each conduction band valley.

edge and the marks are the threshold energy in each direction. Bowing parameter is chosen to be 0.24 from experimental value. ${ }^{9}$ For $\langle 100\rangle$ direction in the unstrained case, the threshold energy exactly follows the band gap for the entire germanium mole fraction range considered. For electrons along the $\langle 111\rangle$ direction, the threshold energy is slightly larger than the band gap and there is no threshold condition from $0 \%$ to $50 \%$ germanium for the $\langle 111\rangle$ direction. In the strained case, it is very important to note that the decrease of the heavy hole effective mass has no effect on the threshold energy because of the fact that final carriers lie approximately at the band edge where the group velocity is almost zero.

Before the calculation of the impact ionization coefficient in $\mathrm{Si}_{1-x} \mathrm{Ge}_{x}$ alloy, Monte Carlo simulation was performed for pure silicon to confirm the scattering parameters. Because the impact ionization rate near the threshold in indirect material is very soft and has a cubic relation dependence ${ }^{10}$ upon $\left(E-E_{\mathrm{tL}}\right)$ rather than square relation like the Keldysh model, ${ }^{11}$ Thoma's model ${ }^{12}$ is used in the calculation to describe the "super soft" region near the threshold. In Fig. 2, the Monte Carlo data are shown along with experimental results. ${ }^{13-15}$ The agreement of the calculated quantities with the corresponding experimental data validates the Monte Carlo method used in this work. Monte Carlo parameters with the changed threshold energy are next applied to

TABLE I. Threshold energy for electron impact ionization in pure silicon and pure germanium. Data in parentheses come from Ref. 6. Units are eV for all the values.

\begin{tabular}{lcc}
\hline \hline & Silicon & Germanium \\
\hline Band gap energy & $1.12(1.1)$ & $0.664(0.7)$ \\
Threshold energy $\langle 100\rangle$ & $1.1223(1.1)$ & $0.8394(0.9)$ \\
Threshold energy $\langle 111\rangle$ & $\cdots$ & $0.8226(0.8)$ \\
\hline \hline
\end{tabular}

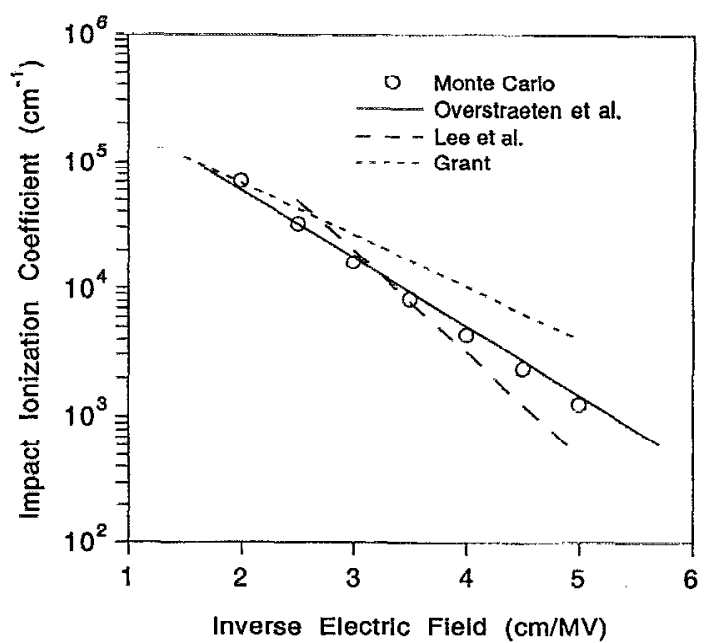

FIG. 2. Electron impact ionization coefficient from Monte Carlo simulations, compared with experimental data (from Refs. 13-15) in pure silicon.

study the impact ionization in $\mathrm{Si}_{1-x} \mathrm{Ge}_{x}$ alloy assuming that they do not change much up to $30 \%$ germanium.

In the $\mathrm{Si}_{1-x} \mathrm{Ge}_{x}$ alloy, an important additional scattering is the alloy scattering. Recently, the alloy scattering has been described by a set of parameters $U_{\text {all }}$ and $r_{0}$ where assuming a perfectly random alloy, it has been found that $U_{\text {all }}=0.7 \mathrm{eV}$ and $r_{0}=\sqrt{3} a / 4(2.36 \AA)$. $^{2}$ The energy-dependent matrix element for alloy scattering has the following form:

$$
M_{k k^{\prime}}=\int_{0}^{r_{0}} e^{-i \mathbf{k}^{t} \cdot \mathbf{r}} U_{\mathrm{all}}(\mathbf{r}) e^{i \mathbf{k} \cdot \mathbf{r}} d \mathbf{r} .
$$

At low electric field, it is reasonable to assume that $q r_{0} \ll 1$ so that

$$
M_{k k^{\prime}} \approx \frac{4}{3} \pi U_{\mathrm{all}} r_{0}^{3}
$$

where

$$
q=\left|\mathbf{k}-\mathbf{k}^{\prime}\right| \text {. }
$$

At high electric field where the term $q r_{0}$ cannot be ignored, the matrix element need to have an accurate form expressed by

$$
M_{k k^{\prime}}=4 \pi U_{\mathrm{all}} \frac{\sin q r_{0}-q r_{0} \cos q r_{0}}{q^{3}}
$$

It is important to point out that at low electric field the transport is governed by $U_{\text {all }}^{2} r_{0}^{6}$, but at high electric field the transport is very much dependent upon $r_{0}$. The value of $r_{0}$ is in general larger than $\sqrt{3} a / 4$ if the alloy is clustered. We will present results for $\alpha$ for unstrained $\mathrm{Si}_{1-x} \mathrm{Ge}_{x}$ with $10 \% \mathrm{Ge}$ using three different values of $U_{\text {all }}$ and $r_{0}$ which are consistent with low field transport.

In Fig. 3, the results of these sets are compared with two extreme cases, i.e., $\alpha$ without alloy scattering and with constant matrix element in Eq. (7). The used values of $U_{\text {all }}$ (and $\left.r_{0}\right)$ are $0.7 \mathrm{eV}(2.36 \AA), 0.6 \mathrm{eV}(2.49 \AA)$, and $0.5 \mathrm{eV}(2.64$ $\AA$ ). The large value of $r_{0}$ reduces the alloy scattering rate and alloy scattering angle. From Fig. 3, it is seen that $\alpha$ ap- 


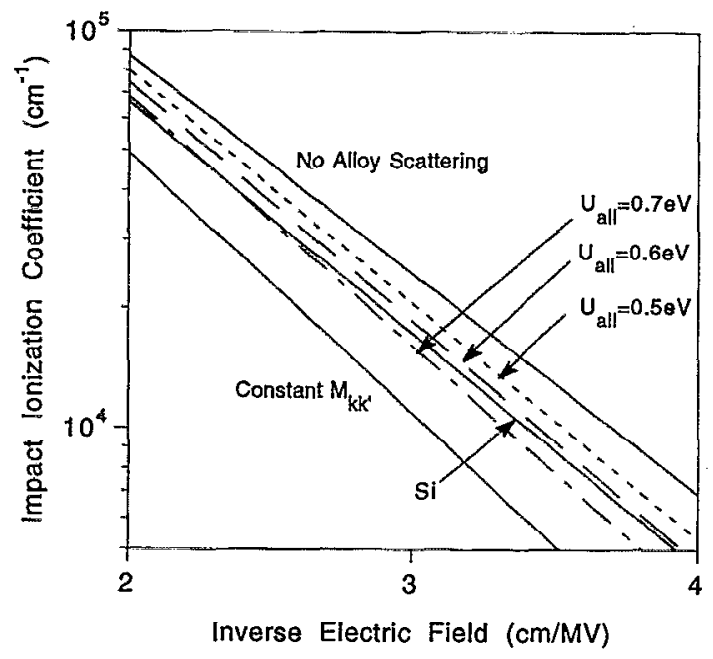

FIG. 3. Electron impact ionization coefficients from different sets of $U_{\text {al }}$ and $r_{0}$. The results are compared with two extreme cases of no alloy scattering and constant matrix element. As $r_{0}$ increases, the effect of alloy scattering is decreased and the result approach to no alloy scattering case.

proaches the no alloy scattering case as $r_{0}$ increases. With $U_{\text {all }}$ value of $0.7 \mathrm{eV}$ which is used for the following Monte Carlo simulations, $\alpha$ is found to be smaller than the value for silicon up to $400 \mathrm{kV} / \mathrm{cm}$. These results show the importance of alloy scattering on the impact ionization coefficient since the threshold energy for silicon and unstrained $\mathrm{Si}_{1-x} \mathrm{Ge}_{x}$ alloy is almost the same.

The calculated impact ionization coefficients in $\mathrm{Si}_{1-x} \mathrm{Ge}_{x}$ alloy up to $30 \% \mathrm{Ge}$ are shown in Fig. 4 for the unstrained and the strained case, respectively. The value used for $U_{\text {all }}$ (and $\left.r_{0}\right)$ is $0.7 \mathrm{eV}(2.36 \AA)$, so that the alloy is assumed not to be clustered. In Fig. 4, the coefficient in the unstrained $\mathrm{Si}_{1-x} \mathrm{Ge}_{x}$ increases slightly as the germanium mole fraction; however, it is smaller than that in pure silicon up to $300-400 \mathrm{kV} / \mathrm{cm}$ due to the effect of alloy scattering and the relatively small change of the threshold energy. On the other hand, the strained system has a much larger impact ionization coefficient. Note that the conduction band edge density of states in the strained $\mathrm{Si}_{1-x} \mathrm{Ge}_{x}$ decreases because the number of band edge valleys decreases from 6 to 4 . This would suggest that the impact ionization coefficient might decrease since the final state density of states decreases rapidly with strain. However, as seen in Fig. 1, the threshold energy decreases rapidly with strain. Thus, the results of Fig. 4 indicate that the most important factor in determining the value of $\alpha$ in the strained $\mathrm{Si}_{1-x} \mathrm{Ge}_{x}$ is the reduced threshold energy rather than the degeneracy lifting at the band edge.

In conclusion, the threshold energy for electron impact ionization is calculated on the basis of the conduction band with nonparabolic ellipsoid and the valence band using $\mathbf{k} \cdot \mathbf{p}$ method. In $X$ valley, the threshold energy follows the bandgap energy while it is slightly larger than band-gap energy in

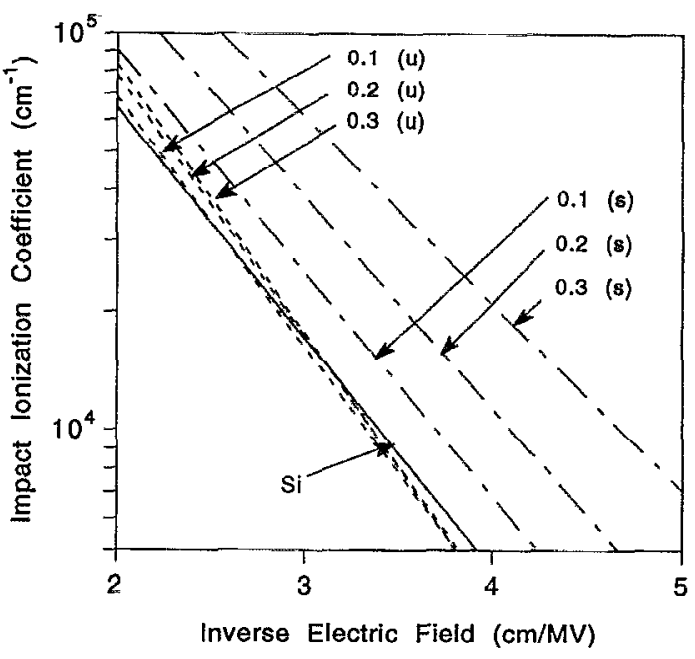

FIG. 4. Monte Carlo simulation results of electron impact ionization coefficients with different germanium mole fraction in unstrained $\mathrm{Si}_{1-x} \mathrm{Ge}_{x}$ and strained $\mathrm{Si}_{1-x} \mathrm{Ge}_{x}$ on $\{100\}$ silicon substrate compared with that in pure silicon. $(u)$ and $(s)$ represent unstrained and strained, respectively.

$L$ valley. It is found that reduced heavy hole effective mass makes no effect on the threshold energy in the strained $\mathrm{Si}_{1-x} \mathrm{Ge}_{x}$. With these results, electron impact ionization coefficient is estimated using Monte Carlo simulation for unstrained $\mathrm{Si}_{1-x} \mathrm{Ge}_{x}$ and strained $\mathrm{Si}_{1-x} \mathrm{Ge}_{x}$ on $\{100\}$ silicon substrate. It is found that the strained $\mathrm{Si}_{1-x} \mathrm{Ge}_{x}$ has larger $\alpha$ than silicon while the unstrained $\mathrm{Si}_{1-x} \mathrm{Ge}_{x}$ has a value comparable to that of silicon.

This work was supported under the Army URI program and under AFOSR Contract No. AFOSR 91-0349.

${ }^{1}$ J. D. Cressler, J. H. Comfort, E. F. Crabbe, G. L. Patton, J. M. C. Stork, J. Sun, and B. S. Meyerson, IEEE Trans. Electron Devices ED-40, 525 (1993).

${ }^{2}$ V. Sankaran, J. M. Hinckley, and J. Singh, IEEE Trans. Electron Devices ED-40, 1589 (1993).

${ }^{3}$ J. C. Bean, L. C. Feldman, A. T. Fiory, S. Nakahara, and I. K. Robinson, J. Vac. Sci. Technol. A 2, 436 (1984).

${ }^{4}$ R. People and J. C. Bean, Appl. Phys. Lett. 47, 322 (1985).

${ }^{5}$ S. F. Nelson, K. Ismail, J. O. Chu, and B. S. Meyerson, Appl. Phys. Lett. 63, 367 (1993).

${ }^{6}$ C. L. Anderson and C. R. Crowell, Phys. Rev. B 5, 6 (1972).

${ }^{7}$ C. Smith and A. D. Welbourn, Proceedings of the 1987 Bipolar Circuits and Technology Meeting, edited by J. Jopke (IEEE, New York, 1987), p. $57-60$.

${ }^{8}$ J. M. Hinckley and J. Singh, Phys. Rev. B 41, 2912 (1990).

${ }^{9}$ J. S. Kline, F. H. Pollak, and M. Cardona, ITelv. Phys. Acta 41, 968 (1968).

${ }^{10}$ A. R. Beattie, Semicond. Sci. Technol. 3, 48 (1988).

${ }^{11}$ L. V. Keldysh, Sov. Phys. JETP 10, 509 (1960).

${ }^{12}$ R. Thoma, H. J. Peifer, W. L. Engl, W. Quade, R. Brunetti, and C. Jacoboni, J. Appl. Phys. 69, 789 (1991).

${ }^{13}$ C. A. Lee, R. A. Logan, R. L. Bartdorf, J. J. Kleimack, and W. Wiegmann, Phys. Rev. A 134, 761 (1964).

${ }^{14} \mathrm{R}$. Van Overstraeten and H. De Man, Solid-State Electron. 13, 583 (1970).

${ }^{15}$ W. N. Grant, Solid-State Electron. 16, 1189 (1973). 